\title{
MFS-based solution to two-dimensional linear thermoelasticity problems
}

\author{
L. Marin ${ }^{1,2}$ \& A. Karageorghis ${ }^{3}$ \\ ${ }^{1}$ Institute of Solid Mechanics, Romanian Academy, Bucharest, Romania \\ ${ }^{2}$ Centre for Continuum Mechanics, Faculty of Mathematics and \\ Computer Science, University of Bucharest, Romania \\ ${ }^{3}$ Department of Mathematics and Statistics, University of Cyprus, \\ Nicosia, Cyprus
}

\begin{abstract}
We propose the numerical approximation of the boundary and internal thermoelastic fields in the case of two-dimensional isotropic linear thermoelastic solids by combining the method of fundamental solutions (MFS) with the method of particular solutions (MPS). A particular solution of the non-homogeneous equations of equilibrium associated with a two-dimensional isotropic linear thermoelastic material is derived based on the MFS approximation of the boundary value problem for the heat conduction equation.

Keywords: linear thermoelasticity, direct problems, method of fundamental solutions, particular solution.
\end{abstract}

\section{Introduction}

The method of fundamental solutions (MFS) is a meshless/meshfree boundary collocation method which is applicable to boundary value problems for which a fundamental solution of the operator in the governing equation is known. In spite of this restriction, the MFS has become very popular primarily because of the ease with which it can be implemented, in particular for problems in complex geometries. Since its introduction as a numerical method by Mathon and Johnston [1], it has been successfully applied to a large variety of physical problems, an account of which may be found in the survey papers [2,3]. 
The MFS, in conjunction with the method of particular solutions (MPS) and the dual reciprocity method, was applied to the numerical solution of direct problems in three-dimensional isotropic linear thermoelasticity by Karageorghis and Smyrlis [4] and Tsai [5], respectively. To the best of our knowledge, direct problems associated with the two-dimensional isotropic linear thermoelasticity have not been solved by the MFS as yet. Therefore, it is the purpose of this study to propose a combined MFS-MPS approach for such problems.

\section{Mathematical formulation}

Consider a domain $\Omega \subset \mathbb{R}^{2}$ which is bounded by a (piecewise) smooth curve $\partial \Omega$ and occupied by an isotropic solid characterised by the thermal conductivity, $\kappa$, the coefficient of linear thermal expansion, $\alpha_{\mathrm{T}}$, Poisson's ratio, $\nu$, and the shear modulus, $G$, respectively.

In the framework of isotropic linear thermoelasticity, the strain tensor, $\boldsymbol{\epsilon}=$ $\left[\epsilon_{i j}\right]_{1 \leq i, j \leq 2}$, is related to the stress tensor, $\sigma=\left[\sigma_{i j}\right]_{1 \leq i, j \leq 2}$, by means of the constitutive law of thermoelasticity, namely

$$
\boldsymbol{\epsilon}(\mathbf{x})=\frac{1}{2 G}\left[\boldsymbol{\sigma}(\mathbf{x})-\frac{\bar{\nu}}{1+\bar{\nu}} \operatorname{tr}(\boldsymbol{\sigma}(\mathbf{x})) \mathbf{I}\right]+\bar{\alpha}_{\mathrm{T}} \mathrm{T}(\mathbf{x}) \mathbf{I}, \quad \mathbf{x} \in \bar{\Omega}=\Omega \cup \partial \Omega .
$$

Here $\mathbf{I}=\left[\delta_{i j}\right]_{1 \leq i, j \leq 2}$ denotes the identity matrix in $\mathbb{R}^{2 \times 2}, \bar{\nu}=\nu$ for a plane strain state and $\bar{\nu}=\nu /(1+\nu)$ for a plane stress state, while $\bar{\alpha}_{\mathrm{T}}=\alpha_{\mathrm{T}}$ and $\bar{\alpha}_{\mathrm{T}}=\alpha_{\mathrm{T}}(1+\nu) /(1+2 \nu)$ in case of the plane strain and plane stress states, respectively. We note from eqn (1) that the shear strains are not affected by the temperature as the free thermal expansion does not produce any angular distortion in an isotropic material. The constitutive law of thermoelasticity (1) can also be conveniently expressed in terms of the stresses as

$$
\boldsymbol{\sigma}(\mathbf{x})=2 G\left[\boldsymbol{\epsilon}(\mathbf{x})+\frac{\bar{\nu}}{1-2 \bar{\nu}} \operatorname{tr}(\boldsymbol{\epsilon}(\mathbf{x})) \mathbf{I}\right]-\bar{\gamma} \mathrm{T}(\mathbf{x}) \mathbf{I}, \quad \mathbf{x} \in \bar{\Omega}
$$

where $\bar{\gamma}=2 G \bar{\alpha}_{\mathrm{T}}(1+\bar{\nu}) /(1-2 \bar{\nu})$.

The equations of equilibrium are similar to those of isotropic linear elasticity since they are based on purely mechanical considerations. The stress tensor can be expressed in terms of the displacement derivatives by combining the constitutive law (2) with the kinematic relation

$$
\boldsymbol{\epsilon}(\mathbf{x})=\frac{1}{2}\left(\nabla \mathbf{u}(\mathbf{x})+\nabla \mathbf{u}(\mathbf{x})^{\top}\right), \quad \mathbf{x} \in \bar{\Omega},
$$

to yield

$$
\boldsymbol{\sigma}(\mathbf{x})=G\left[\left(\nabla \mathbf{u}(\mathbf{x})+\nabla \mathbf{u}(\mathbf{x})^{\top}\right)+\frac{2 \bar{\nu}}{1-2 \bar{\nu}}(\nabla \cdot \mathbf{u}(\mathbf{x})) \mathbf{I}\right]-\bar{\gamma} \mathrm{T}(\mathbf{x}) \mathbf{I}, \quad \mathbf{x} \in \bar{\Omega} .
$$

By assuming the absence of body forces, one obtains the equilibrium equations of isotropic linear thermoelasticity in terms of the displacement vector and 
the temperature (also known as the Navier-Lamé system of isotropic linear thermoelasticity), namely

$$
-\nabla \cdot \boldsymbol{\sigma}(\mathbf{x}) \equiv \mathscr{L} \mathbf{u}(\mathbf{x})+\bar{\gamma} \nabla \mathrm{T}(\mathbf{x})=\mathbf{0}, \quad \mathbf{x} \in \Omega
$$

where $\mathscr{L}=\left(\mathscr{L}_{1}, \mathscr{L}_{2}\right)^{\top}$ is the partial differential operator associated with the Navier-Lamé system of isotropic linear elasticity, i.e.

$$
\mathscr{L} \mathbf{u}(\mathbf{x}) \equiv-G\left[\nabla \cdot\left(\nabla \mathbf{u}(\mathbf{x})+\nabla \mathbf{u}(\mathbf{x})^{\top}\right)+\frac{2 \bar{\nu}}{1-2 \bar{\nu}} \nabla(\nabla \cdot \mathbf{u}(\mathbf{x}))\right], \mathbf{x} \in \Omega
$$

Further, we let $\mathbf{n}(\mathbf{x})$ be the outward unit normal vector at $\partial \Omega$ and $\mathbf{t}(\mathbf{x})$ be the traction vector at $\mathbf{x} \in \partial \Omega$ given by

$$
\mathbf{t}(\mathbf{x}) \equiv \boldsymbol{\sigma}(\mathbf{x}) \mathbf{n}(\mathbf{x}), \quad \mathbf{x} \in \partial \Omega
$$

If the boundary segments $\Gamma_{\mathbf{u}} \subset \partial \Omega$ and $\Gamma_{\mathbf{t}} \subset \partial \Omega$ are such that $\Gamma_{\mathbf{u}} \cap \Gamma_{\mathbf{t}}=\varnothing$ and $\Gamma_{\mathbf{u}} \cup \Gamma_{\mathbf{t}}=\partial \Omega$, and the displacement and traction vectors are prescribed on these boundaries, i.e.

$$
\mathbf{u}(\mathbf{x})=\widetilde{\mathbf{u}}(\mathbf{x}), \quad \mathbf{x} \in \Gamma_{\mathbf{u}}
$$

and

$$
\mathbf{t}(\mathbf{x})=\widetilde{\mathbf{t}}(\mathbf{x}), \quad \mathbf{x} \in \Gamma_{\mathbf{t}}
$$

then eqns (5), (8a) and (8b) represent the boundary value problem associated with the equations of equilibrium for two-dimensional steady-state isotropic linear thermoelasticity.

Next, we let $q(\mathbf{x})$ be the normal heat flux at a point $\mathbf{x} \in \partial \Omega$ defined by

$$
\mathrm{q}(\mathbf{x}) \equiv-(\kappa \nabla \mathrm{T}(\mathbf{x})) \cdot \mathbf{n}(\mathbf{x}), \quad \mathbf{x} \in \partial \Omega
$$

If the temperature and normal heat flux are prescribed on the boundaries $\Gamma_{\mathrm{T}} \subset$ $\partial \Omega$ and $\Gamma_{\mathrm{q}} \subset \partial \Omega$, respectively, where $\Gamma_{\mathrm{T}} \cap \Gamma_{\mathrm{q}}=\varnothing$ and $\Gamma_{\mathrm{T}} \cup \Gamma_{\mathrm{q}}=\partial \Omega$, then one obtains the boundary value problem associated with the heat conduction equation for two-dimensional steady-state isotropic linear thermoelasticity. In the absence of heat sources, this problem can be recast as

$$
\begin{gathered}
-\nabla \cdot(\kappa \nabla \mathrm{T}(\mathbf{x}))=0, \quad \mathbf{x} \in \Omega, \\
\mathrm{T}(\mathbf{x})=\widetilde{\mathrm{T}}(\mathbf{x}), \quad \mathbf{x} \in \Gamma_{\mathrm{T}} \\
\mathrm{q}(\mathbf{x})=\widetilde{\mathrm{q}}(\mathbf{x}), \quad \mathbf{x} \in \Gamma_{\mathrm{q}} .
\end{gathered}
$$




\section{Algorithm}

In this study, we propose the numerical solution of boundary value problems associated with two-dimensional isotropic linear thermoelastic solids, i.e. eqns (5), (8a) and (8b), and eqns (10a)-(10c), respectively, using the MFS in conjunction with the MPS. More precisely, the boundary value problem for the heat conduction equation (10a)-(10c) is first solved numerically by applying a standard MFS. Next, based on this numerical approximation for the temperature field in the solid, we derive - to our knowledge, for the first time - a particular solution of the non-homogeneous equilibrium equations (5). Finally, we apply again a standard MFS to the resulting boundary value problem corresponding to the homogeneous equilibrium equations for a two-dimensional isotropic linear elastic material.

The numerical procedure described above can be summarised as follows:

Step 1. Solve the thermal problem (10a)-(10c) using a standard MFS to determine the unknown boundary temperature $\left.\mathrm{T}\right|_{\Gamma_{\mathrm{q}}}$ and flux $\left.\mathrm{q}\right|_{\Gamma_{\mathrm{T}}}$, as well as the temperature distribution in the domain $\left.\mathrm{T}\right|_{\Omega}$.

Step 2. Determine a particular solution $\mathbf{u}^{(\mathrm{P})}$ of the non-homogeneous equilibrium equations (5) in $\mathbb{R}^{2}$, as well as the corresponding particular strain tensor

$$
\boldsymbol{\epsilon}^{(\mathrm{P})}(\mathbf{x})=\frac{1}{2}\left(\nabla \mathbf{u}^{(\mathrm{P})}(\mathbf{x})+\nabla \mathbf{u}^{(\mathrm{P})}(\mathbf{x})^{\top}\right), \quad \mathbf{x} \in \mathbb{R}^{2},
$$

stress tensor

$$
\boldsymbol{\sigma}^{(\mathrm{P})}(\mathbf{x})=2 G\left[\boldsymbol{\epsilon}^{(\mathrm{P})}(\mathbf{x})+\frac{\bar{\nu}}{1-2 \bar{\nu}} \operatorname{tr}\left(\boldsymbol{\epsilon}^{(\mathrm{P})}(\mathbf{x})\right) \mathbf{I}\right], \quad \mathbf{x} \in \mathbb{R}^{2},
$$

and traction vector

$$
\mathbf{t}^{(\mathrm{P})}(\mathbf{x})=\boldsymbol{\sigma}^{(\mathrm{P})}(\mathbf{x}) \mathbf{n}(\mathbf{x}), \quad \mathbf{x} \in \partial \Omega .
$$

Step 3. Solve the resulting direct problem for the homogeneous equilibrium equations in two-dimensional isotropic linear elasticity, i.e.

$$
\begin{gathered}
\mathscr{L} \mathbf{u}^{(\mathrm{H})}(\mathbf{x})=\mathbf{0}, \quad \mathbf{x} \in \Omega, \\
\mathbf{u}^{(\mathrm{H})}(\mathbf{x})=\widetilde{\mathbf{u}}(\mathbf{x})-\mathbf{u}^{(\mathrm{P})}(\mathbf{x}), \quad \mathbf{x} \in \Gamma_{\mathbf{u}}, \\
\mathbf{t}^{(\mathrm{H})}(\mathbf{x})=\widetilde{\mathbf{t}}(\mathbf{x})-\left[\mathbf{t}^{(\mathrm{P})}(\mathbf{x})-\bar{\gamma} \mathrm{T}(\mathbf{x}) \mathbf{n}(\mathbf{x})\right], \quad \mathbf{x} \in \Gamma_{\mathbf{t}},
\end{gathered}
$$

using a standard MFS to determine the unknown boundary displacement $\left.\mathbf{u}^{(\mathrm{H})}\right|_{\Gamma_{\mathbf{t}}}$ and traction $\left.\mathbf{t}^{(\mathrm{H})}\right|_{\Gamma_{\mathbf{u}}}$, as well as $\left.\mathbf{u}^{(\mathrm{H})}\right|_{\Omega},\left.\boldsymbol{\epsilon}^{(\mathrm{H})}\right|_{\Omega}$ and $\left.\sigma^{(\mathrm{H})}\right|_{\Omega}$.

Step 4. On applying the superposition principle, determine the unknown boundary displacement $\left.\mathbf{u}\right|_{\Gamma_{\mathbf{t}}}=\left.\mathbf{u}^{(\mathrm{H})}\right|_{\Gamma_{\mathbf{t}}}+\left.\mathbf{u}^{(\mathrm{P})}\right|_{\Gamma_{\mathbf{t}}}$ and boundary traction $\left.\mathbf{t}\right|_{\Gamma_{\mathbf{u}}}=\left.\mathbf{t}^{(\mathrm{H})}\right|_{\Gamma_{\mathbf{u}}}+\left.\left(\mathbf{t}^{(\mathrm{P})}-\bar{\gamma} \mathrm{T} \mathbf{n}\right)\right|_{\Gamma_{\mathbf{u}}}$, as well as the 


$$
\begin{aligned}
& \text { mechanical fields inside the domain, namely }\left.\mathbf{u}\right|_{\Omega}=\left.\mathbf{u}^{(\mathrm{H})}\right|_{\Omega}+\left.\mathbf{u}^{(\mathrm{P})}\right|_{\Omega}, \\
& \left.\mathbf{\epsilon}\right|_{\Omega}=\left.\mathbf{\epsilon}^{(\mathrm{H})}\right|_{\Omega}+\left.\boldsymbol{\epsilon}^{(\mathrm{P})}\right|_{\Omega} \text { and }\left.\boldsymbol{\sigma}\right|_{\Omega}=\left.\boldsymbol{\sigma}^{(\mathrm{H})}\right|_{\Omega}+\left.\left(\boldsymbol{\sigma}^{(\mathrm{P})}-\bar{\gamma} \mathrm{T} \mathbf{I}\right)\right|_{\Omega} .
\end{aligned}
$$

\section{Method of fundamental solutions}

Step 1. For the first step of the algorithm described above, consider the fundamental solution, $F$, of the heat balance equation (10a) for two-dimensional steady-state heat conduction in an isotropic homogeneous medium [2], namely

$$
\mathrm{F}(\mathbf{x}, \xi)=-\frac{1}{2 \pi} \log \|\mathbf{x}-\xi\|, \quad \mathbf{x} \in \bar{\Omega},
$$

where $\mathbf{x}=\left(x_{1}, x_{2}\right)$ is a collocation point and $\xi=\left(\xi_{1}, \xi_{2}\right) \in \mathbb{R}^{2} \backslash \bar{\Omega}$ is a singularity or source point. The main idea in the MFS is to approximate the temperature in the solution domain by a linear combination of fundamental solutions with respect to $\mathrm{N}_{\mathrm{s}}^{\mathrm{L}}$ singularities, $\left\{\xi^{(n)}\right\}_{n=1}^{\mathrm{N}_{\mathrm{s}}^{\mathrm{L}}}$, in the form

$$
\mathrm{T}(\mathbf{x}) \approx \mathrm{T}_{\mathrm{N}_{\mathrm{s}}^{\mathrm{L}}}\left(\mathbf{c}^{(1)}, \boldsymbol{\xi} ; \mathbf{x}\right)=\sum_{n=1}^{\mathrm{N}_{\mathrm{s}}^{\mathrm{L}}} \mathrm{c}_{n}^{(1)} \mathrm{F}\left(\mathbf{x}, \xi^{(n)}\right), \mathbf{x} \in \bar{\Omega},
$$

where $\mathbf{c}^{(1)}=\left[\mathrm{c}_{1}^{(1)}, \ldots, \mathrm{c}_{\mathrm{N}_{\mathrm{s}}^{\mathrm{L}}}^{(1)}\right]^{\top} \in \mathbb{R}_{\mathrm{s}}^{\mathrm{N}_{\mathrm{s}}^{\mathrm{L}}}$ and $\xi \in \mathbb{R}^{2 \mathrm{~N}_{\mathrm{s}}^{\mathrm{L}}}$ is a vector containing the coordinates of the singularities $\left\{\xi^{(n)}\right\}_{n=1}^{N_{s}^{L}}$. From equations (9) and (13) it follows that the normal heat flux, through a curve defined by the outward unit normal vector $\mathbf{n}(\mathbf{x})$, can be approximated on the boundary $\partial \Omega$ by

$$
\mathrm{q}(\mathbf{x}) \approx \mathrm{q}_{\mathrm{N}_{\mathrm{s}}^{\mathrm{L}}}\left(\mathbf{c}^{(1)}, \xi ; \mathbf{x}\right)=-\sum_{n=1}^{\mathrm{N}_{\mathrm{s}}^{\mathrm{L}}} \mathrm{c}_{n}^{(1)}\left[\kappa \nabla_{\mathbf{x}} \mathrm{F}\left(\mathbf{x}, \xi^{(n)}\right) \cdot \mathbf{n}(\mathbf{x})\right], \mathbf{x} \in \partial \Omega
$$

Next, we select $\mathrm{N}_{\mathrm{c}}^{\mathrm{T}}$ collocation points, $\left\{\mathbf{x}^{(n)}\right\}_{n=1}^{\mathrm{N}_{\mathrm{c}}^{\mathrm{T}}} \subset \Gamma_{\mathrm{T}}$, and $\mathrm{N}_{\mathrm{c}}^{\mathrm{q}}$ collocation points, $\left\{\mathbf{x}^{\left(\mathrm{N}_{\mathrm{c}}^{\mathrm{T}}+n\right)}\right\}_{n=1}^{\mathrm{N}_{\mathrm{c}}^{\mathrm{q}}} \subset \Gamma_{\mathrm{q}}$, where $\mathrm{N}_{\mathrm{c}}^{\mathrm{T}}+\mathrm{N}_{\mathrm{c}}^{\mathrm{q}}=\mathrm{N}_{\mathrm{c}}^{\mathrm{L}}$, and collocate the boundary conditions (10b) and (10c) to obtain the following system of linear equations with respect to the unknown coefficients $\mathbf{c}^{(1)} \in \mathbb{R}^{\mathrm{N}_{\mathrm{s}}^{\mathrm{L}}}$ :

$$
\mathbf{A}^{(11)} \mathbf{c}^{(1)}=\mathbf{f}^{(1)} .
$$

Here $\mathbf{A}^{(11)} \in \mathbb{R}^{\mathrm{N}_{\mathrm{c}}^{\mathrm{L}} \times \mathrm{N}_{\mathrm{s}}^{\mathrm{L}}}$ is the corresponding matrix whose elements are calculated from eqns (14) and (15), respectively, while $\mathbf{f}^{(1)} \in \mathbb{R}^{\mathrm{N}_{c}^{\mathrm{L}}}$ is the righthand side vector containing the corresponding discretised Dirichlet and Neumann data as given by eqns (10b) and (10c), respectively. 
Step 2. The MFS approximation for the particular solution to the nonhomogeneous equilibrium equations (5) in $\mathbb{R}^{2}$ is given by

$$
\begin{aligned}
& \mathbf{u}^{(\mathrm{P})}(\mathbf{y}) \approx \mathbf{u}_{\mathrm{N}_{\mathrm{s}}^{\mathrm{L}}}^{(\mathrm{P})}\left(\mathbf{c}^{(1)}, \boldsymbol{\xi} ; \mathbf{y}\right) \\
& =-\frac{\bar{\alpha}_{\mathrm{T}}}{4 \pi}\left(\frac{1+\bar{\nu}}{1-\bar{\nu}}\right) \sum_{n=1}^{\mathrm{N}_{\mathrm{s}}^{\mathrm{L}}} \mathrm{c}_{n}^{(1)} \log \left\|\mathbf{y}-\xi^{(n)}\right\|\left(\mathbf{y}-\xi^{(n)}\right), \mathbf{y} \in \mathbb{R}^{2} \backslash \bigcup_{n=1}^{\mathrm{N}_{\mathrm{s}}^{\mathrm{L}}}\left\{\xi^{(n)}\right\} .
\end{aligned}
$$

As a direct consequence of approximation (17), the corresponding particular traction vector on the boundary $\partial \Omega$ is approximated as

$$
\begin{aligned}
& \mathbf{t}^{(\mathrm{P})}(\mathbf{y}) \approx \mathbf{t}_{\mathrm{N}_{\mathrm{s}}^{\mathrm{L}}}^{(\mathrm{P})}\left(\mathbf{c}^{(1)}, \boldsymbol{\xi} ; \mathbf{y}\right) \\
&=-\frac{G \bar{\alpha}_{\mathrm{T}}}{2 \pi}\left(\frac{1+\bar{\nu}}{1-\bar{\nu}}\right) \sum_{n=1}^{\mathrm{N}_{\mathrm{s}}^{\mathrm{L}}} \mathrm{c}_{n}^{(1)}\left[\frac{1}{1-2 \bar{\nu}}\left(\log \left\|\mathbf{y}-\xi^{(n)}\right\|+\bar{\nu}\right) \mathbf{n}(\mathbf{y})\right. \\
&\left.+\frac{\left(\mathbf{y}-\xi^{(n)}\right) \cdot \mathbf{n}(\mathbf{y})}{\left\|\mathbf{y}-\xi^{(n)}\right\|^{2}}\left(\mathbf{y}-\xi^{(n)}\right)\right], \quad \mathbf{y} \in \partial \Omega,
\end{aligned}
$$

while the term $\left(\mathbf{t}^{(\mathrm{P})}-\bar{\gamma} \mathrm{T} \mathbf{n}\right)$ is approximated on $\partial \Omega$ by

$$
\begin{aligned}
\mathbf{t}^{(\mathrm{P})}(\mathbf{y})-\bar{\gamma} \mathrm{T}(\mathbf{y}) \mathbf{n}(\mathbf{y}) \approx \mathbf{t}_{\mathrm{N}_{\mathrm{s}}^{\mathrm{L}}}^{(\mathrm{P})}\left(\mathbf{c}^{(1)}, \boldsymbol{\xi} ; \mathbf{y}\right)-\bar{\gamma} \mathrm{T}_{\mathrm{N}_{\mathrm{s}}^{\mathrm{L}}}(\mathbf{y}) \mathbf{n}(\mathbf{y}) \\
=-\frac{G \bar{\alpha}_{\mathrm{T}}}{2 \pi}\left(\frac{1+\bar{\nu}}{1-\bar{\nu}}\right) \sum_{n=1}^{\mathrm{N}_{\mathrm{s}}^{\mathrm{L}}} \mathrm{c}_{n}^{(1)}\left[\left(\log \left\|\mathbf{y}-\xi^{(n)}\right\|-\frac{\bar{\nu}}{1-2 \bar{\nu}}\right) \mathbf{n}(\mathbf{y})\right. \\
\left.+\frac{\left(\mathbf{y}-\xi^{(n)}\right) \cdot \mathbf{n}(\mathbf{y})}{\left\|\mathbf{y}-\xi^{(n)}\right\|^{2}}\left(\mathbf{y}-\xi^{(n)}\right)\right], \quad \mathbf{y} \in \partial \Omega .
\end{aligned}
$$

Note that once the coefficients, $\mathbf{c}^{(1)} \in \mathbb{R}^{\mathrm{N}_{\mathrm{s}}^{\mathrm{L}}}$, corresponding to the thermal problem (10a)-(10c) are retrieved by solving eqn (16), the particular solutions for the boundary displacement vector on $\Gamma_{\mathbf{t}}$ and boundary traction vector on $\Gamma_{\mathbf{u}}$ are expressed via eqns (17) and (18), respectively.

Step 3. In the case of the Cauchy-Navier system associated with the two-dimensional isotropic linear elasticity, the fundamental solution for the displacement vector, $\mathbf{U}=\left[\mathrm{U}_{i j}\right]_{1 \leq i, j \leq 2}$, is given by [6]

$$
\begin{aligned}
\mathbf{U}_{i j}(\mathbf{y}, \boldsymbol{\eta}) & =\frac{1}{8 \pi G(1-\bar{\nu})}\left[-(3-4 \bar{\nu}) \log \|\mathbf{y}-\boldsymbol{\eta}\| \delta_{i j}\right. \\
& \left.+\frac{y_{i}-\eta_{i}}{\|\mathbf{x}-\boldsymbol{\eta}\|} \frac{y_{j}-\eta_{j}}{\|\mathbf{x}-\boldsymbol{\eta}\|}\right], \quad i, j=1,2,
\end{aligned}
$$

where $\mathbf{y}=\left(y_{1}, y_{2}\right) \in \bar{\Omega}$ is a collocation point, $\boldsymbol{\eta}=\left(\eta_{1}, \eta_{2}\right) \in \mathbb{R}^{2} \backslash \bar{\Omega}$ is a singularity and $\delta_{i j}$ is the Kronecker-delta symbol. On differentiating eqn (16) with 
respect to $y_{k}, k=1,2$, one obtains the derivatives of the fundamental solution for the displacement vector, denoted by $\partial_{y_{k}} \mathrm{U}_{i j}(\mathbf{y}, \boldsymbol{\eta})$, where $\partial_{y_{k}} \equiv \partial / \partial y_{k}$. By combining eqns (8b) and (20), the fundamental solution for the traction vector, $\mathbf{T}=\left[\mathbf{T}_{i j}\right]_{1 \leq i, j \leq 2}$, in the case of two-dimensional isotropic linear elasticity is obtained as [6]

$$
\begin{aligned}
\mathrm{T}_{1 k}(\mathbf{y}, \boldsymbol{\eta}) & =\frac{2 G}{1-2 \bar{\nu}}\left[(1-\bar{\nu}) \partial_{y_{1}} \mathrm{U}_{1 k}(\mathbf{y}, \boldsymbol{\eta})+\bar{\nu} \partial_{y_{2}} \mathrm{U}_{2 k}(\mathbf{y}, \boldsymbol{\eta})\right] n_{1}(\mathbf{y}) \\
& +G\left[\partial_{y_{2}} \mathrm{U}_{1 k}(\mathbf{y}, \boldsymbol{\eta})+\partial_{y_{1}} \mathrm{U}_{2 k}(\mathbf{y}, \boldsymbol{\eta})\right] n_{2}(\mathbf{y}), \quad k=1,2
\end{aligned}
$$

and

$$
\begin{aligned}
& \mathrm{T}_{2 k}(\mathbf{y}, \boldsymbol{\eta})=G\left[\partial_{y_{2}} \mathrm{U}_{1 k}(\mathbf{y}, \boldsymbol{\eta})+\partial_{y_{1}} \mathrm{U}_{2 k}(\mathbf{y}, \boldsymbol{\eta})\right] n_{1}(\mathbf{y}) \\
& +\frac{2 G}{1-2 \bar{\nu}}\left[\bar{\nu} \partial_{y_{1}} \mathrm{U}_{1 k}(\mathbf{y}, \boldsymbol{\eta})+(1-\bar{\nu}) \partial_{y_{2}} \mathrm{U}_{2 k}(\mathbf{y}, \boldsymbol{\eta})\right] n_{2}(\mathbf{y}), \quad k=1,2 .
\end{aligned}
$$

Analogously to the MFS approach for the thermal problem, we now consider $\mathrm{N}_{\mathrm{s}}^{\mathrm{E}}$ singularities, $\left\{\boldsymbol{\eta}^{(n)}\right\}_{n=1}^{\mathrm{N}_{\mathrm{s}}^{\mathrm{E}}}$, and approximate the displacement vector, $\mathbf{u}^{(\mathrm{H})}$, associated with the homogeneous equilibrium equation (12a) in the solution domain by a linear combination of the displacement fundamental solutions (20) with respect to these singularities, i.e.

$$
\mathbf{u}^{(\mathrm{H})}(\mathbf{y}) \approx \mathbf{u}_{\mathrm{N}_{\mathrm{s}}^{\mathrm{E}}}^{(\mathrm{H})}\left(\mathbf{c}^{(2)}, \mathfrak{\eta} ; \mathbf{y}\right)=\sum_{n=1}^{\mathrm{N}_{\mathrm{s}}^{\mathrm{E}}} \mathbf{U}\left(\mathbf{y}, \boldsymbol{\eta}^{(n)}\right) \mathbf{c}_{n}^{(2)}, \quad \mathbf{y} \in \bar{\Omega},
$$

where $\mathbf{c}_{n}^{(2)}=\left[\mathrm{c}_{n ; 1}^{(2)}, \mathrm{c}_{n ; 2}^{(2)}\right]^{\top} \in \mathbb{R}^{2}, 1 \leq n \leq \mathrm{N}_{\mathrm{s}}^{\mathrm{E}}, \mathbf{c}^{(2)}=\left[\left(\mathbf{c}_{1}^{(2)}\right)^{\top}, \ldots,\left(\mathbf{c}_{\mathrm{N}_{\mathrm{s}}^{\mathrm{E}}}^{(2)}\right)^{\top}\right]^{\top}$ $\in \mathbb{R}^{2 \mathrm{~N}_{\mathrm{s}}^{\mathrm{E}}}$ and $\boldsymbol{\eta} \in \mathbb{R}^{2 \mathrm{~N}_{\mathrm{s}}^{\mathrm{E}}}$ is a vector containing the coordinates of the singularities $\left\{\boldsymbol{\eta}^{(n)}\right\}_{n=1}^{N_{\mathrm{s}}^{\mathrm{E}}}$. In a similar manner, the traction vector, $\mathbf{t}^{(\mathrm{H})}$, associated with the homogeneous equilibrium equation (12a) is approximated by a linear combination of the traction fundamental solutions (21), namely

$$
\mathbf{t}^{(\mathrm{H})}(\mathbf{y}) \approx \mathbf{t}_{\mathrm{N}_{\mathrm{s}}^{\mathrm{E}}}^{(\mathrm{H})}\left(\mathbf{c}^{(2)}, \mathbf{\eta} ; \mathbf{y}\right)=\sum_{n=1}^{\mathrm{N}_{\mathrm{s}}^{\mathrm{E}}} \mathbf{T}\left(\mathbf{y}, \boldsymbol{\eta}^{(n)}\right) \mathbf{c}_{n}^{(2)}, \quad \mathbf{y} \in \partial \Omega .
$$

By collocating the boundary conditions (12b) and (12c) at the collocation points $\left\{\mathbf{y}^{(n)}\right\}_{n=1}^{\mathrm{N}_{\mathrm{c}}^{\mathrm{u}}} \subset \Gamma_{\mathbf{u}}$ and $\left\{\mathbf{y}^{\left(\mathrm{N}^{\mathbf{u}}+n\right)}\right\}_{n=1}^{\mathrm{N}_{\mathrm{c}}^{\mathrm{t}}} \subset \Gamma_{\mathbf{t}}$, respectively, where $\mathrm{N}^{\mathbf{u}}+\mathrm{N}^{\mathbf{t}}=\mathrm{N}_{\mathrm{c}}^{\mathrm{E}}$, one obtains the following system of linear equations with respect to the unknown coefficients $\mathbf{c}^{(2)} \in \mathbb{R}^{2 \mathrm{~N}_{\mathrm{s}}^{\mathrm{E}}}$ :

$$
\mathbf{A}^{(22)} \mathbf{c}^{(2)}=\mathbf{f}^{(2)}-\mathbf{A}^{(21)} \mathbf{c}^{(1)} .
$$

Here $\mathbf{A}^{(22)} \in \mathbb{R}^{2 \mathrm{~N}_{c}^{\mathrm{E}} \times 2 \mathrm{~N}_{\mathrm{s}}^{\mathrm{E}}}$ is the corresponding matrix whose elements are calculated from eqns (22) and (23), respectively, $\mathbf{f}^{(2)} \in \mathbb{R}^{2 \mathrm{~N}_{\mathrm{c}}^{\mathrm{E}}}$ is the right-hand 
side vector containing the corresponding discretised Dirichlet and Neumann data as given by eqns (12b) and (12c), respectively, and the elements of the matrix $\mathbf{A}^{(21)} \in \mathbb{R}^{2 \mathrm{~N}_{\mathrm{c}}^{\mathrm{E}} \times \mathrm{N}_{\mathrm{s}}^{\mathrm{L}}}$ are determined from those of the matrices that approximate $\mathbf{u}^{(\mathrm{P})}\left(\mathbf{y}^{(n)}\right)$ and $\left(\mathbf{t}^{(\mathrm{P})}-\bar{\gamma} \mathrm{T} \mathbf{n}\right)\left(\mathbf{y}^{(n)}\right), 1 \leq n \leq \mathrm{N}_{\mathrm{c}}^{\mathrm{E}}$, according to eqns (12a), (12b), (17) and (19).

Step 4. Having determined the coefficients, $\mathbf{c}^{(2)} \in \mathbb{R}^{2 \mathrm{~N}_{\mathrm{s}}^{\mathrm{E}}}$, the approximations of the boundary displacement, $\left.\mathbf{u}\right|_{\Gamma_{\mathbf{t}}}$, and traction vectors, $\left.\mathbf{t}\right|_{\Gamma_{\mathbf{u}}}$, are obtained via the superposition principle and eqns (17), (19), (22) and (23).

It should be mentioned that, in order to uniquely determine the solutions $\mathbf{c}^{(1)} \in \mathbb{R}^{\mathrm{N}_{\mathrm{s}}^{\mathrm{L}}}$ and $\mathbf{c}^{(2)} \in \mathbb{R}^{2 \mathrm{~N}_{\mathrm{s}}^{\mathrm{E}}}$, the corresponding numbers of boundary collocation points and singularities must satisfy the inequalities $\mathrm{N}_{\mathrm{s}}^{\mathrm{L}} \leq \mathrm{N}_{\mathrm{c}}^{\mathrm{L}}$ and $\mathrm{N}_{\mathrm{s}}^{\mathrm{E}} \leq \mathrm{N}_{\mathrm{c}}^{\mathrm{E}}$, respectively. For exact boundary data, these systems can be solved by a direct method, such as the least-squares method or, equivalently, the inversion of the normal equation. However, in the case of perturbed boundary data, such an approach would produce a highly unstable solution and hence systems (16) and (24) should be solved, in a stable manner, by using e.g. the Tikhonov regularization method [7].

\section{Numerical results}

We consider an isotropic linear thermoelastic material which occupies the annular domain $\Omega=\left\{\mathrm{x} \in \mathbb{R}^{2} \mid \mathrm{R}_{\text {int }}<\|\mathrm{x}\|<\mathrm{R}_{\text {out }}\right\}$, where $\mathrm{R}_{\text {int }}=\mathrm{R}_{\text {out }} / 2=1.0$, and is characterised by the following material constants: $G=4.80 \times 10^{10} \mathrm{~N} / \mathrm{m}^{2}$, $\nu=0.34, \kappa=4.01 \mathrm{~W} \mathrm{~m}^{-1} \mathrm{~K}^{-1}$ and $\alpha_{\mathrm{T}}=16.5 \times 10^{-6}{ }^{\circ} \mathrm{C}^{-1}$. We also assume that the thermoelastic fields associated with the material occupying the domain $\Omega$ correspond to constant inner and outer temperatures, $\mathrm{T}_{\text {int }}=\mathrm{T}_{\text {out }} / 2=1{ }^{\circ} \mathrm{C}$, as well as constant inner and outer radial pressures, $\sigma_{\text {int }}=\sigma_{\text {out }} / 2=10^{10} \mathrm{~N} / \mathrm{m}^{2}$, respectively. Such a direct problem admits the following analytical solution:

$$
\begin{aligned}
& \mathrm{T}^{(\text {an })}(\mathbf{x})=\mathrm{T}_{\text {out }} \frac{\log \left(\|\mathbf{x}\| / \mathrm{R}_{\text {int }}\right)}{\log \left(\mathrm{R}_{\text {out }} / \mathrm{R}_{\text {int }}\right)}+\mathrm{T}_{\text {int }} \frac{\log \left(\mathrm{R}_{\text {out }} /\|\mathbf{x}\|\right)}{\log \left(\mathrm{R}_{\text {out }} / \mathrm{R}_{\text {int }}\right)}, \quad \mathbf{x} \in \bar{\Omega}, \\
& \mathrm{q}^{(\mathrm{an})}(\mathbf{x})=-\kappa \frac{\mathrm{T}_{\text {out }}-\mathrm{T}_{\text {int }}}{\log \left(\mathrm{R}_{\text {out }} / \mathrm{R}_{\text {int }}\right)} \frac{\mathbf{x} \cdot \mathbf{n}(\mathbf{x})}{\|\mathbf{x}\|^{2}}, \quad \mathbf{x} \in \partial \Omega, \\
& \mathbf{u}^{(\mathrm{an})}(\mathbf{x})=\left[\frac{\bar{\alpha}_{\mathrm{T}}}{2} \frac{1+\bar{\nu}}{1-\bar{\nu}} \frac{\mathrm{T}_{\text {out }}-\mathrm{T}_{\text {int }}}{\log \left(R_{\text {out }} / R_{\text {int }}\right)} \log \|\mathbf{x}\|\right. \\
& \left.+\frac{1}{2 G}\left(\mathrm{~V} \frac{1-\bar{\nu}}{1+\bar{\nu}}+\mathrm{W} \frac{1}{\|\mathbf{x}\|^{2}}\right)\right] \mathbf{x}, \quad \mathbf{x} \in \bar{\Omega}, \\
& \mathbf{t}^{(\mathrm{an})}(\mathbf{x})= \begin{cases}-\sigma_{\text {out }} \mathbf{n}(\mathbf{x}), & \mathbf{x} \in \Gamma_{\text {out }} \equiv\left\{\mathbf{x} \in \partial \Omega \mid\|\mathbf{x}\|=\mathrm{R}_{\text {out }}\right\} \\
-\sigma_{\text {int }} \mathbf{n}(\mathbf{x}), & \mathbf{x} \in \Gamma_{\text {int }} \equiv\left\{\mathbf{x} \in \partial \Omega \mid\|\mathbf{x}\|=\mathrm{R}_{\text {int }}\right\}\end{cases}
\end{aligned}
$$


where

$$
\begin{gathered}
\mathrm{V} \equiv-\frac{\sigma_{\text {out }}^{(\mathrm{H})} \mathrm{R}_{\text {out }}^{2}-\sigma_{\text {int }}^{(\mathrm{H})} \mathrm{R}_{\text {int }}^{2}}{\mathrm{R}_{\text {out }}^{2}-\mathrm{R}_{\text {int }}^{2}}, \quad \mathrm{~W} \equiv \frac{\left(\sigma_{\text {out }}^{(\mathrm{H})}-\sigma_{\text {int }}^{(\mathrm{H})}\right) \mathrm{R}_{\text {out }}^{2} \mathrm{R}_{\text {int }}^{2}}{\mathrm{R}_{\text {out }}^{2}-\mathrm{R}_{\text {int }}^{2}}, \\
\sigma_{\text {out }}^{(\mathrm{H})} \equiv \sigma_{\text {out }}-\bar{\gamma} \mathrm{T}_{\text {out }}+G \bar{\alpha}_{\mathrm{T}} \frac{1+\bar{\nu}}{1-2 \bar{\nu}}\left(\frac{1}{1-\bar{\nu}} \log \mathrm{R}_{\text {out }}+1\right),
\end{gathered}
$$

and

$$
\sigma_{\text {int }}^{(\mathrm{H})} \equiv \sigma_{\text {int }}-\bar{\gamma} \mathrm{T}_{\text {int }}+G \bar{\alpha}_{\mathrm{T}} \frac{1+\bar{\nu}}{1-2 \bar{\nu}}\left(\frac{1}{1-\bar{\nu}} \log \mathrm{R}_{\text {int }}+1\right) .
$$

In this study, we have considered $\mathrm{N}_{\mathrm{c}}^{\mathrm{L}}=\mathrm{N}_{\mathrm{c}}^{\mathrm{E}}=\mathrm{N}$ collocation points on $\partial \Omega=$ $\Gamma_{\text {out }} \cup \Gamma_{\text {int }}$, such that $2 \mathrm{~N} / 3$ and $\mathrm{N} / 3$ uniformly distributed collocation points have been taken on the outer $\Gamma_{\text {out }}$ and inner boundaries $\Gamma_{\text {int }}$, respectively. According to the notations used in Section 4, we have $\mathrm{N}_{\mathrm{c}}^{\mathrm{T}}=\mathrm{N}_{\mathrm{c}}^{\mathrm{t}}=\mathrm{N}$ and $\mathrm{N}_{\mathrm{c}}^{\mathrm{q}}=\mathrm{N}_{\mathrm{c}}^{\mathrm{u}}=0$. We have also considered $\mathrm{N}_{\mathrm{s}}^{\mathrm{L}}=\mathrm{N}_{\mathrm{s}}^{\mathrm{E}}=\mathrm{M}$ uniformly distributed singularities corresponding to the outer ( $2 \mathrm{M} / 3$ singularities) and the inner boundaries $(\mathrm{M} / 3$ singularities), which are preassigned and kept fixed throughout the solution process on the pseudo-boundaries $\widetilde{\Gamma}_{\text {out }}=\left\{\mathrm{x} \in \mathbb{R}^{2} \mid\|\mathbf{x}\|=\mathrm{R}_{\text {out }}+\mathrm{d}_{1}\right\}$ and $\widetilde{\Gamma}_{\text {int }}=\left\{\mathrm{x} \in \mathbb{R}^{2} \mid\|\mathrm{x}\|=\mathrm{R}_{\text {int }}-\mathrm{d}_{2}\right\}$, respectively, where $0.05 \leq \mathrm{d}_{1} \leq 1.0$ and $0.02 \leq \mathrm{d}_{2} \leq 0.9$.

In order to asses the accuracy and convergence of the proposed MFS approach, for any real-valued function $f: \partial \Omega \longrightarrow \mathbb{R}$, we define the corresponding pointwise normalised error of $f$ at $\mathbf{x} \in \partial \Omega$ and the maximum normalised error of $f$ on $\partial \Omega$ by

$$
\operatorname{err}(f(\mathbf{x}))=\frac{\left|f^{(\text {num })}(\mathbf{x})-f(\mathbf{x})\right|}{\max _{\mathbf{y} \in \partial \Omega}|f(\mathbf{y})|}, \quad \mathbf{x} \in \partial \Omega,
$$

and

$$
\operatorname{Err}(f)=\max _{\mathbf{x} \in \partial \Omega} \operatorname{err}(f(\mathbf{x})),
$$

respectively, where $f^{(\mathrm{num})}(\mathbf{x})$ denotes an approximate numerical value for $f(\mathbf{x})$, $\mathbf{x} \in \partial \Omega$. Analogously, one can define the pointwise normalised error of $f$ at $\mathbf{x} \in \Omega$ and the maximum normalised error of $f$ inside the domain $\Omega$.

Figs. 1(a)-(d) present the numerical results for the $x_{1}$-component of the displacement on the boundary $\partial \Omega$, in terms of the maximum normalised error $\operatorname{Err}\left(u_{1}\right)$ defined by eqn $(27 b)$, obtained using the proposed approach, various numbers of boundary collocation points and singularities, i.e. $M=N \in$ $\{48,60,72,84\}, \mathrm{d}_{1} \in[0.05,1.0]$ and $\mathrm{d}_{2} \in[0.02,0.9]$. It can be seen from these figures that the numerical solution for the displacement $\left.u_{1}\right|_{\partial \Omega}$ is an accurate and convergent approximation to its corresponding exact solution with respect to increasing the number of collocation points, as well as the distances to the pseudo-boundaries $\widetilde{\Gamma}_{\text {out }}$ and $\widetilde{\Gamma}_{\text {int }}$, respectively. Although not presented, it is 
reported that similar results have been obtained for the $x_{2}$-component of the displacement, traction vector, temperature and normal heat flux on $\partial \Omega$, as well as the displacement vector, stress tensor and temperature field inside the domain $\Omega$.



(a) $\mathrm{M}=\mathrm{N}=48$

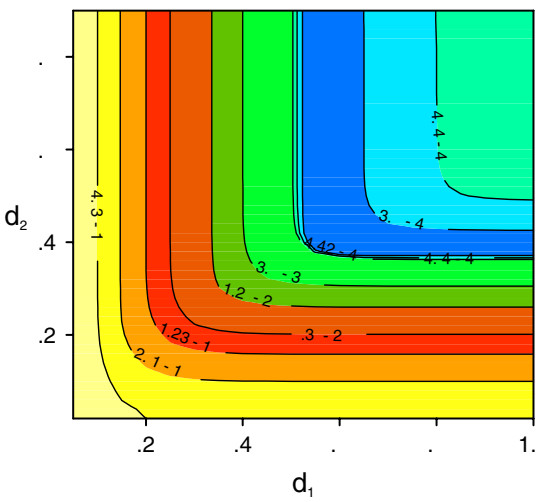

(c) $\mathrm{M}=\mathrm{N}=72$

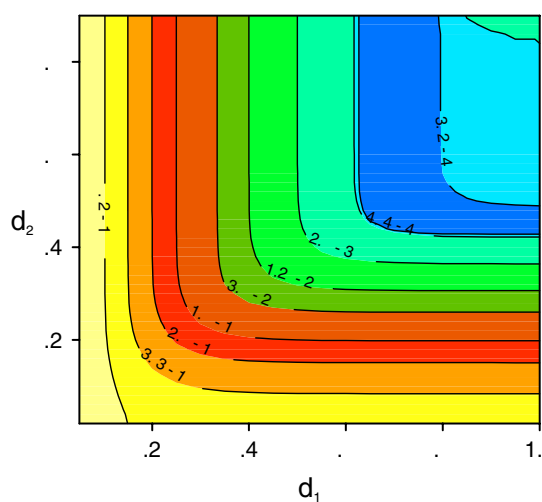

(b) $\mathrm{M}=\mathrm{N}=60$

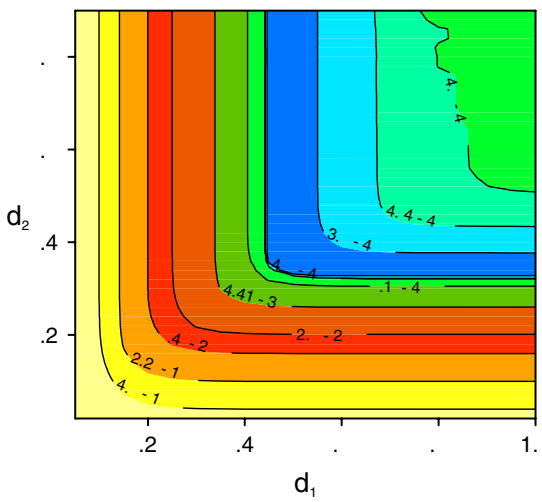

(d) $\mathrm{M}=\mathrm{N}=84$

Figure 1: The maximum normalised error $\operatorname{Err}\left(\mathrm{u}_{1}\right)$ on $\partial \Omega$ as a function of the distances $\mathrm{d}_{1}$ and $\mathrm{d}_{2}$, obtained using various numbers of collocation points and sources, namely (a) $\mathrm{M}=\mathrm{N}=48$, (b) $\mathrm{M}=\mathrm{N}=60$, (c) $\mathrm{M}=\mathrm{N}=72$ and (d) $\mathrm{M}=\mathrm{N}=84$. 


\section{Conclusions}

In this paper, the solution of direct problems in two-dimensional linear isotropic thermoelasticity was investigated using the MFS in conjunction with the MPS. This approach is based on the development of a novel particular solution of the non-homogeneous equations of equilibrium associated with a two-dimensional isotropic linear thermoelastic material which depends entirely on the MFS approximation of the boundary value problem for the heat conduction equation. The numerical results obtained show the convergence and accuracy of the proposed method.

\section{Acknowledgement}

The financial support received from the Romanian National Authority for Scientific Research (CNCS-UEFISCDI), project number PN-II-ID-PCE-2011$3-0521$, is gratefully acknowledged.

\section{References}

[1] Mathon, R. and Johnston, R. L., The approximate solution of elliptic boundary value problems by fundamental solutions. SIAM Journal on Numerical Analysis, 14, pp. 638-650, 1977.

[2] Fairweather, G. and Karageorghis, A., The method of fundamental solutions for elliptic boundary value problems. Advances in Computational Mathematics, 9, pp. 69-95, 1998.

[3] Fairweather, G., Karageorghis, A. and Martin, P. A., The method of fundamental solutions for scattering and radiation problems. Engineering Analysis with Boundary Elements, 27, pp. 759-769, 2003.

[4] Karageorghis, A. and Smyrlis, Y.-S., Matrix decomposition MFS algorithms for elasticity and thermo-elasticity problems in axisymmetric domains. Journal of Computational and Applied Mathematics, 206, pp. 774-795, 2007.

[5] Tsai, C. C., The method of fundamental solutions with dual reciprocity for three-dimensional thermoelasticity under arbitrary forces. International Journal for Computer-Aided Engineering and Software, 26, pp. 229-244, 2009.

[6] Aliabadi, M. H., The Boundary Element Method. Applications in Solids and Structures. John Wiley \& Sons: London, 2002. Volume 2.

[7] Tikhonov, A. N. and Arsenin, V. Y., Methods for Solving Ill-Posed Problems. Nauka: Moscow, 1986. 